\title{
Reference frame consistency in CHAMP and GRACE Earth Gravity Models
}

\author{
Christopher Kotsakis \\ Department of Geodesy and Surveying \\ School of Engineering \\ Aristotle University of Thessaloniki \\ Univ. Box 440, Thessaloniki 54124, Greece \\ Email: kotsaki@topo.auth.gr
}

\begin{abstract}
After the launch of the CHAMP and GRACE satellite missions, an increasing number of spherical harmonic models have become available for the long-, medium- and short-wavelength mapping of the Earth's gravitational field. In view of the need for a coherent comparison between such Earth Gravity Models (EGMs) and a detailed evaluation of their accuracy for various gravity field functionals, it is important to investigate the consistency of their inherent reference frames, especially when their use is intended for high precision studies. Following the methodology described in an earlier paper by Kleusberg (1980), the Helmert transformation parameters among the inherent reference frames for several CHAMP and GRACE models are estimated in this paper. In particular, the differences between the corresponding spherical harmonic coefficients for a given pair of EGMs are parameterized through a 3D similarity transformation model, whose weighted least-squares adjustment yields valuable information for the origin stability, the orientation consistency and the spatial scale variation between their underlying reference frames.
\end{abstract}

Keywords. GRACE, CHAMP, reference frame, spherical harmonics, Helmert transformation

\section{Introduction}

All gravity field functionals obtained from a global Earth Gravity Model (EGM) depend on the terrestrial reference frame that ensues from implementing an optimal inversion procedure to various data sets which are used for the model's development. In the case of processing satellite tracking data, for example, such EGM-related global reference frames can be realized through an appropriate set of constraints that is applied to a number of satellite tracking stations in order to remove the rank deficiency of the input data that lead to the estimation of the lowdegree EGM coefficients; see Pavlis (1998). This occurs if in the processing of the satellite tracking data one solves simultaneously for the tracking sta- tion positions (and possibly velocities) along with the geopotential harmonic coefficients. Alternatively, one may decide to adopt some existing ITRF-type frame to fix a-priori the EGM's reference system, and then process the tracking data (and other types of geodetic observables coming from dedicated satellite gravity missions such as CHAMP or GRACE) exclusively for geopotential recovery. In any case, if we want to maintain a coherent framework for the comparison of current and future EGMs, and to evaluate their prediction accuracy for gravity field functionals, it is important to investigate the consistency of their inherent reference frames.

Compared to the standard direct approach followed by the IERS for the realization of the International Terrestrial Reference Frame (ITRF) in terms of recurring estimates for the $3 \mathrm{D}$ geocentric Cartesian coordinates at a number of control stations (Altamimi et al. 2002), an EGM solution carries through its harmonic coefficients (including the conventionally fixed or estimated zero- and firstdegree coefficients, and its associated scaling factors $G M$ and $a$ ) all the required information to constrain the origin, orientation and scale of a spatial reference system. Different EGMs provide alternative 'indirect realizations' of a 3D Cartesian coordinate system, whose transformation parameters are estimable from the differences of their corresponding spherical harmonic coefficients (Kleusberg 1980).

The consistency of the global reference frames inherent in different EGMs is a critical issue that affects several tasks, including the determination of combined estimates for gravity field signals by merging information from different geopotential models, the comparison and accuracy evaluation of different EGMs, and the calibration of their error models using terrestrial and/or satellite data. Moreover, the origin, orientation and scale stability among the EGMs' reference frames is crucial for estimating and properly monitoring Earth change parameters (e.g. mean sea level changes, Earth rota- 
tion variations, mass re-distribution, etc.) through global gravity field mapping, as well as for integrating geometric and 'gravimetric' reference systems and data sets that are linked to them.

There are several investigations in the geodetic literature which have focused on reference frame consistency aspects in global geopotential models (e.g. Anderle 1974, Rapp and Rummel 1976, Grappo 1980, Lachapelle and Kouba 1981, Kirby and Featherstone 1997). Despite the wide-ranging interpretations of their results, most of these studies have relied on a common methodology, notably the comparison of geometrically derived (i.e. through satellite positioning techniques) and EGM-based geoid undulations over a global, continental or regional network of leveling benchmarks.

The main drawback of such evaluation techniques is that the reference frame transformation parameters (usually three translation components and one scale change factor are only considered), which are obtained from the comparison of geometric and EGM-based geoid heights, are inevitably distorted due to the high correlation caused by the non-uniform distribution and limited spatial coverage of the control points. Even in cases with a truly global and uniform distribution of data points (Schaab and Groten 1979), the conclusions drawn from such an evaluation scheme are likely to be obscured by the coupling of long-wavelength biases from the EGM-geoid heights into the estimates of the reference frame transformation parameters; see Weigel (1993).

Furthermore, the geoid height is a totally insensitive variable to the rotation of the underlying reference system about the symmetry axis of the adopted reference ellipsoid. Hence, it is impossible to estimate this particular datum parameter solely from the differences $N^{G P S}-N^{E G M}$. The latter are able to recover only variations in the direction of the mean Earth rotation axis that is implied in each geoid type, and not orientation differences in the zeromeridian planes of their associated reference frames.

The objective of the present paper is to implement an alternative approach for testing the consistency of the reference frames inherent in different EGMs. The formulation given in Kleusberg (1980) for the Helmert spatial transformation of a spherical harmonic series is employed to estimate the origin, orientation and scale variations among the global reference frames implied in recent CHAMP and GRACE geopotential models. The benefit of such an approach is that it does not involve the intermediate computation and comparison of other gravity field functionals (e.g. geoid heights), but it relies entirely on the original harmonic coefficients of the Earth's gravitational potential that are provided by the specific EGMs under comparison.

\section{Methodology}

Our study is based on the mathematical model given by Kleusberg (1980) for the linear approximation of the Helmert (or 3D Euclidean similarity) transformation for the Earth's gravitational potential harmonic coefficients, under translations, rotations and scale variation in the underlying Cartesian coordinate system. Although the linearized transformation formulae derived in that study are theoretically less rigorous than other versions that appeared in previous references (e.g. Goldstein 1984), they are fairly precise for practical use in global geodetic studies where small (differential) reference frame perturbations are typically involved (Pavlis 1998).

Due to the limited extent of the paper, only a brief overview of the Helmert transformation formulae for spherical harmonic coefficients will be given, without presenting any additional mathematical details or derivations; for a complete description, see the original paper by Kleusberg (1980).

Let us consider the usual expansion of the Earth's gravitational potential in terms of a spherical harmonic series (Heiskanen and Moritz 1967)

$$
V(r, \lambda, \varphi)=\frac{G M}{r} \sum_{n, m}\left(\frac{a}{r}\right)^{n}\left\{\begin{array}{c}
\bar{C}_{n m} \cos m \lambda \\
+ \\
\bar{S}_{n m} \sin m \lambda
\end{array}\right\} \bar{P}_{n m}(\sin \varphi)(1)
$$

The set $\left\{\bar{C}_{n m}, \bar{S}_{n m}\right\}$ contains unitless, fullynormalized spherical harmonic coefficients that are obtained from a global EGM up to a maximum degree of expansion $n_{\max }$. The quantities $G M$ and $a$ correspond to the geocentric gravitational constant and mean equatorial radius, which are the basic scaling factors that are conventionally associated with an EGM solution, while $\bar{P}_{n m}(\cdot)$ represent the fully-normalized associated Legendre functions of degree $n$ and order $m$.

In most EGMs the zero-degree normalized coefficient $\bar{C}_{0,0}$ is usually treated as an errorless quantity, with its value fixed a-priori to 1 . Based on (1), such a constraint gives an initial noise-free approximation for the Earth's gravitational potential

$V(r, \lambda, \varphi) \approx \frac{G M}{r} \bar{C}_{0,0}=\frac{G M}{r}$

which corresponds to a point-mass (or homogeneous sphere) Earth model, with the origin of the EGM's reference frame located at the Earth's center of mass (geocenter). Note that in several geopotential models (e.g. GRIM5, GFZ96, TUM1S, TUM2S) the zero-degree coefficient is not conventionally 
fixed to 1 , and its actual value is associated with a formal statistical error that corresponds to the uncertainty of the geocentric gravitational 'constant' $G M$ which is estimated anew within the EGM development process. In such cases, the following formula relates the accuracy of the zero-degree coefficient with the uncertainty of the associated value for the geocentric gravitational constant

$\sigma_{\bar{C}_{0,0}}=\frac{\sigma_{G M}}{G M}$

The first-degree normalized coefficients $\bar{C}_{1,0}$, $\bar{C}_{1,1}, \bar{S}_{1,1}$ are physically related to the Cartesian shifts of the origin of the EGM's reference frame (in which the geodetic coordinates $r, \lambda, \varphi$ should refer to) with respect to the geocenter. In fact, we have the well-known relationships (e.g. Kirby and Featherstone 1997)

$$
\bar{C}_{1,0}=\frac{z_{c m}}{a \sqrt{3}}, \quad \bar{C}_{1,1}=\frac{x_{c m}}{a \sqrt{3}}, \bar{S}_{1,1}=\frac{y_{c m}}{a \sqrt{3}}
$$

where $x_{c m}, y_{c m}, z_{c m}$ are the Cartesian coordinates of the geocenter with respect to the reference frame associated with the series expansion in (1).

The first-degree coefficients in most EGMs are conventionally fixed to zero, thus enforcing a geocentricity constraint for the global reference system that should be used for the computation of the Earth's gravitational potential (and its functionals) from a spherical harmonic expansion. In several recent CHAMP and GRACE models non-zero estimates and formal error variances are provided for their first-degree coefficients, which are handled as additional unknown parameters within the EGM development process. The resulting Cartesian coordinates for the Earth's geocenter and their accuracy level, with respect to the inherent reference frames for these geopotential models, are given in Table 1.

Table 1. Geocenter's Cartesian coordinates (and their formal accuracy level) with respect to the inherent reference frames in recent CHAMP/GRACE models (in $\mathrm{mm}$ ).

\begin{tabular}{lccc}
\hline Model & $x_{c m}$ & $y_{c m}$ & $z_{c m}$ \\
\hline EIGEN-CG03C & $-5.5 \pm 4.8$ & $-3.4 \pm 4.8$ & $-1.5 \pm 4.4$ \\
EIGEN-CG01C & $-3.8 \pm 6.1$ & $1.2 \pm 6.1$ & $-1.2 \pm 5.5$ \\
EIGEN-CHAMP03S & $-2.7 \pm 3.3$ & $6.1 \pm 3.3$ & $-9.2 \pm 3.4$ \\
TUM2S & $0.2 \pm 0.2$ & $0.7 \pm 0.2$ & $0.2 \pm 0.2$ \\
TUMIS & $-1.1 \pm 0.2$ & $-2.1 \pm 0.2$ & $-15.1 \pm 0.2$ \\
\hline
\end{tabular}

In general, the expansion of the Earth's gravitational potential $V(\cdot)$ in a different reference frame than the one implied in (1) takes the form
$V\left(r^{\prime}, \lambda^{\prime}, \varphi^{\prime}\right)=\frac{G M}{r^{\prime}} \sum_{n, m}\left(\frac{a}{r^{\prime}}\right)^{n}\left\{\begin{array}{c}\bar{C}_{n m}^{\prime} \cos m \lambda^{\prime} \\ + \\ \bar{S}_{n m}^{\prime} \sin m \lambda^{\prime}\end{array}\right\} \bar{P}_{n m}\left(\sin \varphi^{\prime}\right)$

where $r^{\prime}, \lambda^{\prime}, \varphi^{\prime}$ are the coordinates of the evaluation point with respect to a new Cartesian terrestrial coordinate system. Note that the scaling factors $G M$ and $a$ are assumed to retain their conventional values in both reference systems.

Let us assume that the two reference frames differ according to the standard linearized Helmert transformation model (e.g. Soler 1998)

$$
\left[\begin{array}{c}
x^{\prime}-x \\
y^{\prime}-y \\
z^{\prime}-z
\end{array}\right]=\left[\begin{array}{c}
t_{x} \\
t_{y} \\
t_{z}
\end{array}\right]+\left[\begin{array}{ccc}
\delta s & \varepsilon_{z} & -\varepsilon_{y} \\
-\varepsilon_{z} & \delta s & \varepsilon_{x} \\
\varepsilon_{y} & -\varepsilon_{x} & \delta s
\end{array}\right]\left[\begin{array}{l}
x \\
y \\
z
\end{array}\right]
$$

where $t_{x}, t_{y}, t_{z}$ are the translation parameters between the two frames, $\varepsilon_{x}, \varepsilon_{y}, \varepsilon_{z}$ are the rotation angles about the axes of the first frame (anticlockwise rotations assumed positive), and $\delta s$ corresponds to the unitless factor of their differential spatial scale change.

Based on the Cartesian coordinate transformation in (6) and the fact that the gravitational potential $V(\cdot)$ is independent of the reference coordinate system in which we choose to perform its evaluation through a spherical harmonic series, a set of linearized transformation formulae can be derived between the coefficients $\left\{\bar{C}_{n m}, \bar{S}_{n m}\right\}$ and $\left\{\bar{C}_{n m}^{\prime}, \bar{S}_{n m}^{\prime}\right\}$. These formulae were originally published by Kleusberg (1980), and they can be expressed in terms of the general equations

$$
\begin{aligned}
& \bar{C}_{n m}^{\prime}-\bar{C}_{n m}=\delta \bar{C}_{n m}\left(t_{x}\right)+\delta \bar{C}_{n m}\left(t_{y}\right) \\
& +\delta \bar{C}_{n m}\left(t_{z}\right)+\delta \bar{C}_{n m}\left(\varepsilon_{x}\right)+\delta \bar{C}_{n m}\left(\varepsilon_{y}\right) \\
& +\delta \bar{C}_{n m}\left(\varepsilon_{z}\right)+\delta \bar{C}_{n m}(\delta s)
\end{aligned}
$$

and

$\bar{S}_{n m}^{\prime}-\bar{S}_{n m}=\delta \bar{S}_{n m}\left(t_{x}\right)+\delta \bar{S}_{n m}\left(t_{y}\right)$

$+\delta \bar{S}_{n m}\left(t_{z}\right)+\delta \bar{S}_{n m}\left(\varepsilon_{x}\right)+\delta \bar{S}_{n m}\left(\varepsilon_{y}\right)$

$+\delta \bar{S}_{n m}\left(\varepsilon_{z}\right)+\delta \bar{S}_{n m}(\delta s)$

The analytical expressions of the above equations are rather cumbersome, requiring more than one page for their detailed writing, and they will not be given here. In general, for each harmonic coefficient $\bar{C}_{n m}$ or $\bar{S}_{n m}$ being transformed, the righthand side of the above equations depends on the 
values of the 'nearby' coefficients $\bar{C}_{n-1, m+1}$, $\bar{C}_{n, m-1}, \quad \bar{C}_{n-1, m-1}, \quad \bar{C}_{n, m+1}, \quad \bar{C}_{n-1, m}, \bar{S}_{n-1, m+1}$, $\bar{S}_{n, m-1}, \bar{S}_{n-1, m-1}, \bar{S}_{n, m+1}$ and $\bar{S}_{n-1, m}$; for more details, see Kleusberg (1980).

Different EGMs are not always compatible with the same numerical values for their associated scaling factors $G M$ and $a$ (see Table 2). In order to evaluate the consistency of the reference frames inherent in different EGMs through a least-squares (LS) inversion of Kleusberg's transformation model, a re-scaling of their original spherical harmonic coefficients may be needed so that they refer to the same conventional values for the geocentric gravitational constant and mean equatorial radius.

Using as reference the $G M$ and $a$ values associated with the first model $\left\{\bar{C}_{n m}, \bar{S}_{n m}\right\}$, the required re-scaling for the coefficients of the second model has the form

$\left\{\begin{array}{c}\bar{C}_{n m}^{\prime} \\ \bar{S}_{n m}^{\prime}\end{array}\right\}=\frac{G M^{\prime}}{G M}\left(\frac{a^{\prime}}{a}\right)^{n}\left\{\begin{array}{c}\bar{C}_{n m}^{\prime}(\text { orig }) \\ \bar{S}_{n m}^{\prime}(\text { orig })\end{array}\right\}$

where $G M^{\prime}$ and $a^{\prime}$ are the scaling factors associated with the original harmonic coefficients of the second model.

The coefficients $\left\{\bar{C}_{n m}^{\prime}, \bar{S}_{n m}^{\prime}\right\}$ computed from (9) are compatible with the values $G M$ and $a$ associated with $\left\{\bar{C}_{n m}, \bar{S}_{n m}\right\}$ and they should be used, instead of $\left\{\bar{C}_{n m}^{\prime}\right.$ (orig), $\bar{S}_{n m}^{\prime}$ (orig) $\}$, in the formulation of Kleusberg's transformation model (which actually requires that both models refer to the same conventional scaling factors).

\section{Least-squares estimation using Kleusberg's $\left\{\bar{C}_{n m}, \bar{S}_{n m}\right\} \rightarrow\left\{\bar{C}_{n m}^{\prime}, \bar{S}_{n m}^{\prime}\right\}$ transformation model}

For an arbitrary pair of EGMs, a linear system of equations can be formed based on Kleusberg's (1980) transformation model

$\mathbf{y}=\mathbf{A} \mathbf{x}+\mathbf{v}$

where $\mathbf{y}$ is a vector that contains the differences $\bar{C}_{n m}^{\prime}-\bar{C}_{n m}$ and $\bar{S}_{n m}^{\prime}-\bar{S}_{n m}$ of their corresponding harmonic coefficients, $\mathbf{x}$ is an unknown vector with the Helmert transformation parameters of their underlying reference frames, $\mathbf{A}$ is a full-rank design matrix whose elements depend on the harmonic coefficients $\left\{\bar{C}_{n m}, \bar{S}_{n m}\right\}$ of the first 'reference' $\mathrm{EGM}$, and $\mathbf{v}$ is a residual vector due to non-datumrelated errors in the coefficients of both EGMs.

In our study, the vector $\mathbf{x}$ of the Helmert transformation parameters is estimated through a weighted LS adjustment of (10), using the following guidelines:

- the differences according to (7) and (8) are formed for the entire harmonic spectral bandwidth $n, m=0-70$. If the zero- and firstdegree coefficients of both EGMs under comparison are a-priori fixed to 1 and 0 , respectively, then the first four elements of the observation vector $\mathbf{y}$ will be zero;

- a diagonal weight matrix $\mathbf{P}$ is used for the LS adjustment. The weight for each 'observation' $\bar{C}_{n m}^{\prime}-\bar{C}_{n m}$ and $\bar{S}_{n m}^{\prime}-\bar{S}_{n m}$ is taken as

$$
p_{i}=\frac{1}{\sigma_{\bar{C}_{n m}^{\prime}}^{2}+\sigma_{\bar{C}_{n m}}^{2}}
$$

and

$$
p_{i}=\frac{1}{\sigma_{\bar{S}_{n m}^{\prime}}^{2}+\sigma_{\bar{S}_{n m}}^{2}}
$$

respectively. In order to assign realistic error variances to the zero- and first-degree harmonic coefficients (in cases of EGMs where these coefficients are a-priori fixed to the error-free values 1 and 0 , respectively), the following conventions have been adopted.

\section{Conventional (a-priori) EGM 'geocentricity' accuracy level:}

$$
\sigma_{x_{c m}}=\sigma_{y_{c m}}=\sigma_{z_{c m}}=0.01 \mathrm{~m}
$$

from which we can infer the uncertainty in the EGM's first-degree coefficients as

$\sigma_{\bar{C}_{1,0}}=\sigma_{z_{c m}} /(a \sqrt{3})$

$\sigma_{\bar{C}_{1,1}}=\sigma_{x_{c m}} /(a \sqrt{3})$

$\sigma_{\bar{S}_{1,1}}=\sigma_{y_{c m}} /(a \sqrt{3})$

Conventional (a-priori) 'GM' uncertainty level (IERS Conventions, McCarthy and Petit 2004):

$\sigma_{G M}=0.8 \times 10^{6} \mathrm{~m}^{3} \mathrm{~s}^{-2}$ 
from which we can infer the uncertainty in the EGM's zero-degree coefficient as

$\sigma_{\bar{C}_{0,0}}=\sigma_{G M} / G M$

Note that the above conventional scheme for assigning error variances to the zero- and first-degree harmonic coefficients is employed only for EGMs that use the a-priori assumptions $\bar{C}_{0,0}=1$ and/or $\bar{C}_{1,0}=\bar{C}_{1,1}=\bar{S}_{1,1}=0$. For EGMs which are accompanied by non-zero error variances for their zero- and/or first-degree harmonics, the weight determination according to (11) employs the formal error variances of these coefficients, as given in their official release.

\section{Numerical results}

Ten different CHAMP/GRACE EGMs have been used for our numerical tests, which are listed in Table 2 along with their associated scaling factors $G M$ and $a$. Their spherical harmonic coefficients and the corresponding error variances have been obtained from the website of the International Centre of Global Earth Models (ICGEM) at the GeoForschungsZentrum (GFZ), Potsdam (http:// icgem.gfz-otsdam.de/ICGEM/ICGEM.html). In the case where both formal and calibrated error variances are provided for a particular EGM (e.g. EI$G E N-G R A C E 02 S$ ), the latter have been adopted for the statistical weight determination according to (11). It should be pointed out that all tested EGMs are expressed in the tide-free system.

$E I G E N-C G 03 C$ is adopted as the 'reference' model linked to the $\left\{\bar{C}_{n m}, \bar{S}_{n m}\right\}$ coefficients that appear in Kleusberg's transformation model; see (7) and (8). The estimated Helmert parameters will thus be consistent with the transformation scheme GRF1 $\rightarrow G R F 2$, where GRF1 is the inherent reference frame of $E I G E N-C G 03 C$, and GRF2 is the inherent reference frame of every other EGM that is tested.

Hence, the ingoing values $\left\{\bar{C}_{n m}, \bar{S}_{n m}\right\}$ for computing the observation vector $\mathbf{y}$ in (10) will always correspond to the original harmonic coefficients of EIGEN-CG03C, whereas the ingoing values $\left\{\bar{C}_{n m}^{\prime}, \bar{S}_{n m}^{\prime}\right\}$ are obtained by applying the rescaling formula (9) to the original harmonic coefficients of every other EGM to be tested. Based on our particular selection of EGMs (see Table 2) such a re-scaling is necessary only for $T U M 2 S$ and TUM1S.
An example of the correlation matrix for the estimated transformation parameters from the leastsquares adjustment of Kleusberg's model is given in Table 3, for the comparison between EIGEN$C G 03 C$ and $E I G E N-C G 01 C$. It is seen that the correlations between all possible combinations of the Helmert transformation parameters is essentially negligible, thus suggesting a well-conditioned adjustment model. Note that similar patterns of statistical correlation have been obtained in all the adjustment tests that were performed for our study.

Table 2. The conventional values for the geocentric gravitational constant $(G M)$ and the mean equatorial radius $(a)$ associated with the EGMs that are used for the numerical tests in this paper.

\begin{tabular}{lccc}
\hline Model & GM(in $\left.\mathrm{m}^{3} \mathrm{~s}^{-2}\right)$ & $a$ (in m) & $n_{\max }$ \\
\hline EIGEN-CG03C & $398600.4415 \times 10^{9}$ & 6378136.46 & 360 \\
EIGEN-CG01C & $398600.4415 \times 10^{9}$ & 6378136.46 & 360 \\
EIGEN-GL04C & $398600.4415 \times 10^{9}$ & 6378136.46 & 360 \\
EIGEN-CHAMP03S & $398600.4415 \times 10^{9}$ & 6378136.46 & 140 \\
EIGEN-GRACE02S & $398600.4415 \times 10^{9}$ & 6378136.46 & 150 \\
EIGEN-GRACE01S & $398600.4415 \times 10^{9}$ & 6378136.46 & 140 \\
TUM2S & $398600.4418 \times 10^{9}$ & 6378137.00 & 60 \\
TUMIS & $398600.4360 \times 10^{9}$ & 6378137.00 & 60 \\
EIGEN2 & $398600.4415 \times 10^{9}$ & 6378136.46 & 140 \\
EIGENIS & $398600.4415 \times 10^{9}$ & 6378136.46 & 119 \\
\hline
\end{tabular}

The results for the estimated transformation parameters, along with their formal statistical accuracy ( $1 \sigma$ uncertainty level), are given in Table 4 . Based on the values given in this table, the following comments can be made:

- the inherent reference frames of the tested EGMs are consistent, in terms of their origin position, at the level of $1-2 \mathrm{~cm}$;

- an interesting result is the evident bias in the $t_{z}$ values, compared to the equatorial translation components $t_{x}$ and $t_{y}$. A similar 'z-shift' effect has been also reported in other studies on direct comparisons between global terrestrial reference frames (Soler and van Gelder 1987, Boucher and Altamimi 2001, Heflin et al. 2002); see also Schaab and Groten (1979).

- in terms of orientation stability for the mean Earth rotation axis (i.e. rotation angles $\varepsilon_{x}$ and $\varepsilon_{y}$ ), the associated reference frames of the tested EGMs exhibit variations in the order of $10^{-2}-10^{-3}$ arcsec. On the other hand, the rotation angle about the mean Earth rotation axis $\left(\varepsilon_{z}\right)$ appears to have much larger values (by 1-3 orders of magnitude, up to a few arcsec!), a fact that suggests the existence of possible systematic differences in the definition and realization of the zero-meridian plane within the EGMs' reference frames; 
Table 3. Correlation matrix for the estimated transformation parameters between the inherent reference frames in the $E I$ $G E N-C G 03 C$ and $E I G E N-C G 01 C$ models, as obtained from the least-squares adjustment using Kleusberg's (1980) model.

\begin{tabular}{cccccccc}
\hline & $t_{x}$ & $t_{y}$ & $t_{z}$ & $\varepsilon_{x}$ & $\varepsilon_{y}$ & $\varepsilon_{z}$ & $\delta s$ \\
\hline$t_{x}$ & 1.0000000 & 0.0000082 & 0.0000013 & 0.0000312 & -0.0000625 & -0.0000929 & 0.0013781 \\
$t_{y}$ & & 1.0000000 & -0.0000037 & 0.0004224 & -0.0000699 & -0.0044572 & -0.0002247 \\
$t_{y}$ & & & 1.0000000 & 0.0001308 & 0.0011952 & 0.0007413 & 0.0008539 \\
$\varepsilon_{x}$ & & & & 1.0000000 & -0.0012470 & -0.0020342 & -0.0001379 \\
$\varepsilon_{y}$ & & & & & 1.0000000 & 0.0028387 & -0.0000411 \\
$\varepsilon_{z}$ & & & & & & 1.0000000 & -0.00000483 \\
$\delta s$ & & & & & & & \\
\hline
\end{tabular}

- the reference frames associated with most of the tested EGMs show a scale stability at the ppb level, or better. Notable exceptions are the models TUM1S and EIGEN2, where the estimated scale factor reaches the values of -16.21 $\mathrm{ppb}$ and $8.34 \mathrm{ppb}$, respectively. Note that the harmonic coefficients of each tested model have been properly re-scaled before the adjustment so that they refer to the $G M$ and $a$ values of the reference model $E I G E N-C G 03 C$. Thus, the estimated parameter $\delta s$ given in Table 4 is not influenced by any artificial scaling differences that may exist among the harmonic coefficients of the tested EGMs.

The square roots of the a-posteriori variance factors obtained from the previous adjustment tests are shown in Table 5. In most cases their values are close to 1 , indicating that the choice of the diagonal weight matrix $\mathbf{P}$ which was formed according to (7) and (8) is fairly realistic. The most notable exceptions occur for the adjustment tests performed with the TUM1S and TUM2S models, the results of which suggest that the original error variances for their harmonic coefficients are rather optimistic. On the other hand, the test results obtained with $E I$ GEN-GLO4C, EIGEN-CG01C and EIGEN$G R A C E 01 S$ show that their harmonic coefficients were probably assigned a lower statistical weight than they deserve, within the least-squares adjustment of Kleusberg's transformation model.

Table 5. Square root of the a-posteriori variance factor for the least-squares adjustments corresponding to the cases shown in Table 4.

\begin{tabular}{l|c}
\hline Model & $\hat{\sigma}_{\mathrm{o}}$ \\
\hline EIGEN-GL04C & 0.77 \\
EIGEN-CG01C & 0.78 \\
EIGEN-CHAMP03S & 1.60 \\
TUM2S & 2.99 \\
TUM1S & 2.64 \\
EIGEN-GRACE02S & 1.01 \\
EIGEN-GRACE01S & 0.73 \\
EIGEN2 & 1.27 \\
EIGEN1S & 1.87 \\
\hline
\end{tabular}

Table 4. Helmert transformation parameters of the inherent reference frames in CHAMP/GRACE EGMs (with respect to EIGEN-CG03). The differences of spherical harmonic coefficients with their error variances up to $n_{\max }=70$ have been used, including the zero- and first-degree harmonics from all models.

\begin{tabular}{l|ccc|ccc|c}
\hline Model & $t_{x}(\mathrm{~mm})$ & $t_{y}(\mathrm{~mm})$ & $t_{z}(\mathrm{~mm})$ & $\varepsilon_{x}(\mathrm{mas})$ & $\varepsilon_{y}(\mathrm{mas})$ & $\varepsilon_{z}(\mathrm{mas})$ \\
\hline EIGEN-GL04C & $6.3 \pm 8.5$ & $-0.3 \pm 8.5$ & $14.1 \pm 8.3$ & $5.19 \pm 3.75$ & $-0.93 \pm 3.73$ & $-71.07 \pm 53.34$ & $0.59 \pm 2.18$ \\
EIGEN-CG01C & $2.1 \pm 6.0$ & $2.2 \pm 6.0$ & $4.2 \pm 5.5$ & $-0.67 \pm 4.11$ & $-2.82 \pm 4.09$ & $95.21 \pm 61.40$ \\
EIGEN-CHAMP03S & $2.7 \pm 9.4$ & $1.0 \pm 9.3$ & $5.6 \pm 8.8$ & $15.98 \pm 22.18$ & $20.52 \pm 22.02$ & $-582.88 \pm 356.97$ & $0.42 \pm 2.21$ \\
TUM2S ${ }^{(*)}$ & $5.7 \pm 14.5$ & $0.7 \pm 14.5$ & $15.3 \pm 13.1$ & $7.91 \pm 15.45$ & $-4.07 \pm 15.29$ & $-5380.54 \pm 1239.92$ & $1.55 \pm 4.53$ \\
TUMIS & $4.3 \pm 12.8$ & $-1.1 \pm 12.8$ & $-0.9 \pm 11.5$ & $109.53 \pm 15.84$ & $49.15 \pm 15.74$ & $-4099.31 \pm 1306.46$ & $-16.21 \pm 5.28$ \\
EIGEN-GRACE02S & $6.6 \pm 11.0$ & $2.6 \pm 11.0$ & $7.6 \pm 10.6$ & $-4.04 \pm 6.66$ & $-7.18 \pm 6.59$ & $-15.60 \pm 98.64$ \\
EIGEN-GRACE01S & $5.6 \pm 8.1$ & $0.6 \pm 8.1$ & $15.7 \pm 8.0$ & $5.02 \pm 27.52$ & $-5.63 \pm 27.56$ & $-69.55 \pm 217.89$ \\
EIGEN2 & $0.3 \pm 14.1$ & $2.7 \pm 14.1$ & $-11.4 \pm 13.9$ & $10.76 \pm 5.16$ & $27.92 \pm 5.14$ & $1681.47 \pm 253.13$ & $0.01 \pm 2.85$ \\
EIGENIS & $5.4 \pm 20.8$ & $0.3 \pm 20.8$ & $15.1 \pm 20.4$ & $15.94 \pm 77.52$ & $22.30 \pm 85.41$ & $1766.71 \pm 2444.75$ & $0.04 \pm 3.59$ \\
\hline
\end{tabular}

${ }^{(*)}$ The spherical harmonic coefficients with their error variances up to $n_{\max }=60$ have been used for testing these models. 


\section{Conclusions}

Using Kleusberg's (1980) linearized model for the Helmert transformation of the spherical harmonic coefficients of the Earth's gravitational potential, a number of CHAMP/GRACE global geopotential models have been tested in terms of their reference frame consistency. Compared to other techniques that have been used for similar purposes, our methodology relies on the direct adjustment of the differences $\left\{\bar{C}_{n m}^{\prime}-\bar{C}_{n m}\right\}$ and $\left\{\bar{S}_{n m}^{\prime}-\bar{S}_{n m}\right\}$ according to a similarity-type transformation model that relates the underlying EGMs' reference frames, without computing other intermediate gravity field functionals (e.g. geoid heights). It is hoped that the results obtained from this study will be of interest for many gravity field specialists and EGM development teams for the purpose of identifying possible discrepancies in the physical/mathematical models and/or data sets used in the compilation of modern global geopotential models.

Acknowledgements. The author would like to thank Nikos Pavlis for his useful remarks, and also to acknowledge the two anonymous reviewers for their comments.

\section{References}

Altamimi Z, Sillard P, Boucher C (2002) ITRF2000: A new release of the International Terrestrial Reference Frame for earth science applications. J Geoph Res, 107(B10): 2214-2232.

Anderle RJ (1974) Transformation of terrestrial survey data to Doppler satellite datum. J Geoph Res, 79(B35): 5319-5331.

Boucher C, Altamimi Z (2001) ITRS, PZ-90 and WGS84: current realizations and the related transformation parameters. J Geod, 75(11): 613-619.

Goldstein JD (1984) The effect of coordinate system rotations on spherical harmonic expansions. J Geoph Res, 89(B6): 4413-4418.
Grappo GA (1980) Determination of the earth's mean equatorial radius and center of mass from Dopplerderived and gravimetric geoid heights. Manusc Geod, 5(3/4): 201-216.

Heflin M, Argus D, Jefferson D, Webb F, Zumberge J (2002) Comparison of a GPS-defined global reference frame with ITRF2000. GPS Sol, 6(1/2): 72-75.

Heiskanen W, Moritz H (1967) Physical geodesy. WH Freeman, San Francisco.

Kirby JF, Featherstone WE (1997) A study of zero- and first-degree terms in geopotential models in Australia. Geomatics Research Australasia, 66: 93-108.

Kleusberg A (1980) The similarity transformation of the gravitational potential close to the identity. Manusc Geod, 5: 241-256.

Lachapelle G, Kouba J (1981) Relationship between terrestrial and satellite Doppler systems. In: Reference Coordinate Systems for Earth Dynamics (Gaposchkin EM, Kolaczek B, eds.), Reidel, Dordrecht, Holland, pp. 195-203.

McCarthy DD, Petit G (eds., 2004) IERS Conventions 2003. International Earth Rotation and Reference Systems Service (IERS) Technical Note, No. 32, Verlag des Bundesamts für Kartographie und Geodäsie, Frankfurt am Main

Pavlis EC (1998) On the reference frames inherent in recent geopotential models. In: Vermeer M, Adam J (eds.) Proceedings of the Second Continental Workshop on the Geoid in Europe, Budapest, Hungary March 10-14, 1998. Reports of the Finnish Geodetic Institute, Massala, 98:4, pp. 29-40.

Rapp RH, Rummel R (1976) Comparison of Doppler derived undulations with gravimetric undulations considering the zero-order undulation of the geoid. In: Proceedings of the First International Geodetic Symposium on Satellite Doppler Positioning. Defence Mapping Agency, Washington, DC, pp. 389-397.

Schaab H, Groten E (1979) Comparison of geocentric origins of global systems from uniformly distributed data. Bull Geod, 53(1): 11-17.

Soler T, van Gelder BHW (1987) On differential scale changes and the satellite Doppler system z-shift. Geophys J R Astr Soc, 91(3): 639-656.

Soler T (1998) A compendium of transformation formulas useful in GPS work. J Geod, 72(7/8): 482-490.

Weigel G (1993) Geoid undulation computations at Doppler satellite tracking stations. Manusc Geod, 18(1): 10-25. 